\title{
Assessment of the Catford drum in visual acuity testing and its use as a measurement of visual performance in low-vision patients
}

\author{
J J BOOP, J T W VAN DALEN, AND G S TYNER
}

From the Department of Ophthalmology and Visual Sciences, Texas Tech University Health Sciences Center School of Medicine, Lubbock, Texas 79430, USA

SUMMARY Objective measurements of visual acuity were determined with the Catford drum in 82 eyes of patients in our Low-Vision Clinic who typically suffered from visual loss due to macular disease. The results were compared with subjective measurements of visual acuity by the Snellen chart. The findings indicated a significant overestimation of Snellen visual acuities by the Catford drum in $90.2 \%$ of eyes tested by a factor of 1.05 to 20.0 , average 4.73 . The correlation coefficient for the study was $+\mathbf{0 . 4 0}$. This differs from the original results of Catford and Oliver in 1971. In addition, the Catford drum was used on follow-up visits in the same patients to assess 'visual performance'. The initial results showed an improvement in visual acuities when the Catford drum was used in 12 of 15 patients, while the Snellen acuities remained stable when retested after one month of basic instruction and use of standard low-vision aids. This improvement in 'Catford' acuity was by a factor of $0 \cdot 3$ to $10 \cdot 0$, average 4.08 . This is thought to represent the patient's ability to learn the use of eccentric viewing or parafoveal retinal areas for vision. It confirms previous intuitive findings and helps to explain why low-vision patients seem to function at a higher level than expected from their Snellen visual acuities.

Many of our Low-Vision Clinic patients surprise us by the fact that their daily life performance is often so much better than might be expected on the basis of their Snellen visual acuity. Our current method of assessing visual acuity and presumably performance in low-vision patients is by means of a static subjective test, the Snellen chart.

We believe that in addition to the tested Snellen visual acuity fraction every patient has a certain level of 'visual performance' independent of this, which as we define it relates to the patient's ability to function and perform daily life tasks. Furthermore, visual performance is dynamic in that learning and improvement can occur. The majority of our lowvision patients have visual loss secondary to some form of macular disease with normal functioning parafoveal retinal areas. We believe that it is this region of normal surrounding retina and the patient's ability to learn the use of eccentric viewing or fixation

Correspondence to J T W van Dalen, MD, Department of Ophthalmology, Texas Tech University Health Sciences Center, Lubbock, Texas 79430, USA. which allows a higher level of functioning and performance. However, an exact determination of visual performance is a difficult task for which no rapid and simple test has been devised. It is hoped the Catford drum may be useful in evaluating this aspect. In our Low-Vision Clinic we are testing it as a means of estimating visual performance and the ability to learn the use of parafoveal retinal areas for vision.

Studies have been done comparing objective visual acuities with subjective measurements of visual acuities. These studies have used various methods of either inducing or arresting optokinetic nystagmus to determine measurements of objective acuity. A summary of these results reported by Pearson' shows that the correlation coefficient between objective and subjective measurement of visual acuity varied from +0.33 to +0.92 . In defining the correlation coefficient linear regression techniques were used to minimise the sum of the squares of the deviations of the actual data points from the straight line of best fit. In particular, two studies concluded that there are differences in comparing results based on the visual 
acuity being tested. Wolin and Dillman ${ }^{2}$ and Millodot et al. ${ }^{3}$ agreed that for a subject with relatively good acuity the subjective acuity is found to be slightly better than the measured objective acuity. However, the opposite is true for a patient with relatively poor visual acuity.

Catford and Oliver ${ }^{45}$ reported a good correlation in their results between Catford drum acuities and Snellen acuities in adults with visual acuities better than $6 / 60$. The calculated correlation coefficient for that study is $+0 \cdot 87$. Two studies have been published since then which directly compare the use of the Catford drum as an objective measurement of visual acuity with that of Snellen visual acuity. Atkinson et $a l .{ }^{6}$ found that for emmetropes there was an overestimation of visual acuity with the Catford drum by a factor of approximately 4 . For those individuals with reduced vision, either uncorrected myopes or emmetropes viewing through plus lenses to reduce acuity, the ratio between acuity measures involved an overestimation of Snellen acuities by a factor of at least 10 . Khan et al. ${ }^{7}$ reported that eyes with relatively good vision, 20/50 or better, showed a good correlation between the subjective and objective acuities. However, there was poor correlation in eyes with $20 / 60$ vision or less, in which case the objective acuities were generally better than the subjective acuities. Their calculated correlation coefficient was $+0 \cdot 61$.

In summary, it appears that objective acuity measures correlate differently with subjective acuity measures depending on the method used. In dealing specifically with the Catford drum the evidence seems to indicate that the objective acuities as measured by the drum not only generally overestimate the subjective acuities, but do so by a factor which varies with the level of vision.

\section{Materials and methods}

The Catford drum (Fig. 1) was introduced by Catford and Oliver in 1971 as an instrument to determine visual acuity, especially in young children under 3 years of age, when reliable subjective responses cannot be elicited. It provides an objective method of determining visual acuity based on inducing an optokinetic nystagmus. The Catford drum consists of a motor driven drum with separated black dots of various sizes on a white background projected through a central aperture, measuring $4 \times 6 \mathrm{~cm}$. These dots can be rotated from left to right and back to the left again in a continuously repeating manner. The patient is seated the recommended $60 \mathrm{~cm}$ away from the Catford drum and is instructed to watch the selected dot with the eye to be tested. The visual acuity is determined by reducing the target size until

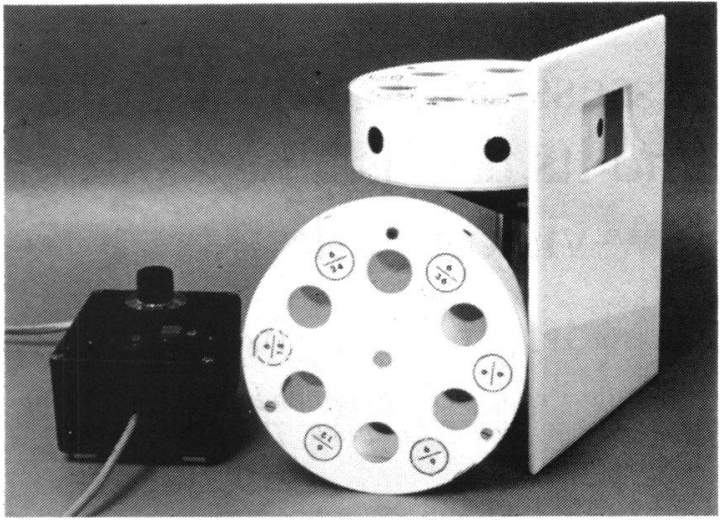

Fig. 1 The Catford drum apparatus.

the smallest dot is found which no longer produces a regular oscillatory eye movement (an optokinetic nystagmus). This endpoint is recorded and accepted as the objective equivalent to the Snellen visual acuity.

Catford and Oliver have calibrated the drum in terms of Snellen acuities from 20/20 to 20/600 based on the dot size at $60 \mathrm{~cm}$, subtending an angle equal to the corresponding Snellen letter at $6 \mathrm{~m}$. The speed of the rotating dot is controlled by a rheostat on an electrical transformer. This was adjusted to obtain the recommended setting allowing a full oscillatory cycle to repeat every one second.

Visual acuity was also measured by a standard Snellen chart projected on a screen at a distance of $6 \mathrm{~m}$. All testing was performed with best spectacle correction. The same room was used for testing of all subjects to allow consistent and reproducible testing conditions. Recommended testing conditions for the Catford drum suggest its use in uniform bright daylight or equivalent artificial light. Uniform illumination was provided by ceiling based fluorescent lights. The luminance as measured at the central aperture of the Catford drum located $60 \mathrm{~cm}$ from the patient was $179 \mathrm{~cd} / \mathrm{m}^{2}$ as measured with a Spectra Pritchard photometer (Model 1980A-PL).

In the first part of the study patients in our LowVision Clinic were tested with the Catford drum at 60 $\mathrm{cm}$ as outlined above to determine objective visual acuity. The same eyes were then tested with the standard Snellen chart at $6 \mathrm{~m}$ to determine subjective visual acuity. The results were then compared. The patients had varying levels of subjective visual acuity from 20/30 to hand motion. In this half of the study only patients who recorded a visual acuity of $20 / 400$ or better were accepted, since this is the upper limit of accuracy for the Snellen chart at $6 \mathrm{~m}$. A total of 82 eyes were tested in this initial study.

In the second part of the study we wanted to assess 


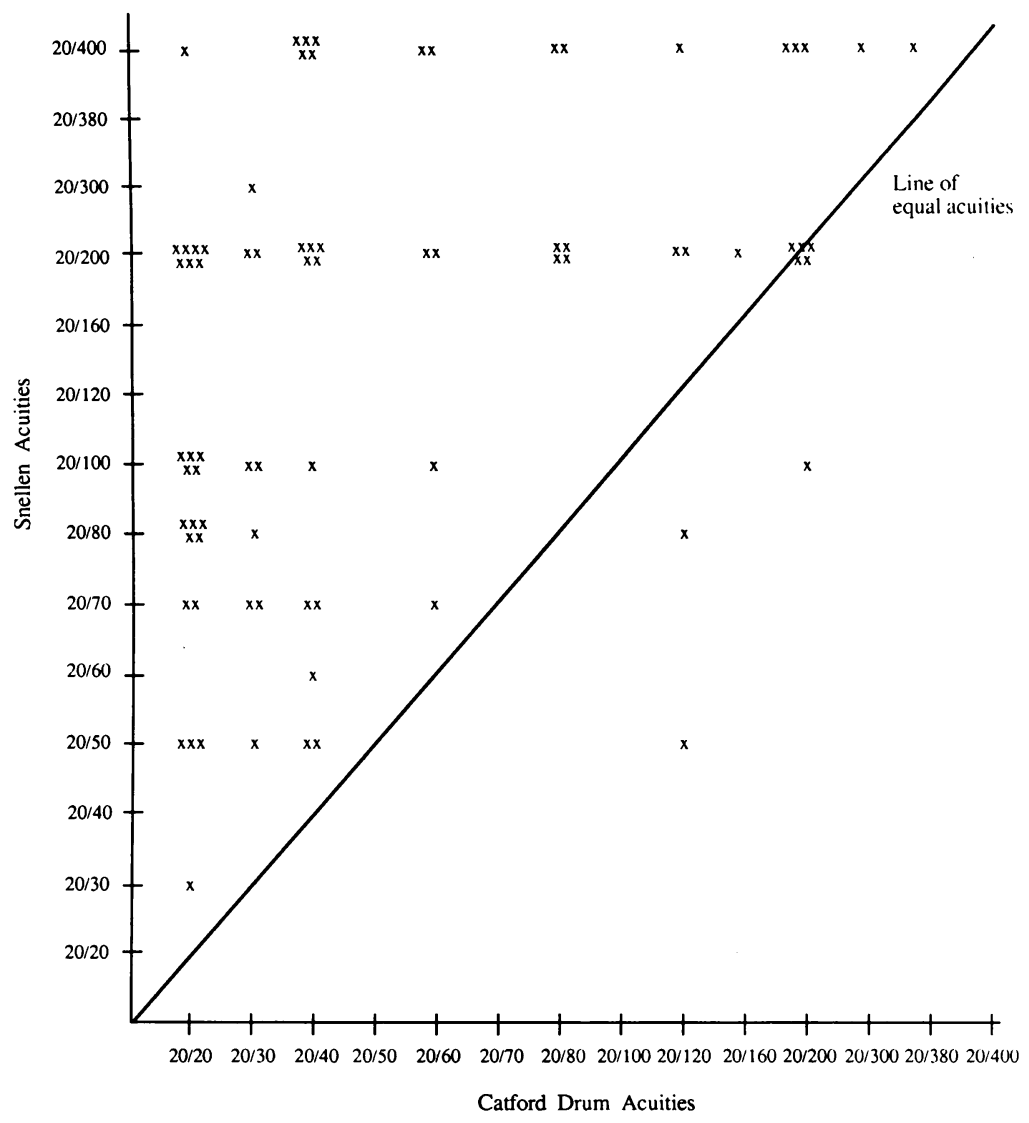

Fig. 2 Display of the Snellen visual acuity and the Catford drum acuity obtained for each eye tested on the initial visit. Each $\mathrm{x}$ represents one eye, with a total of 82 eyes tested.

visual performance as related to the patient's ability to learn the use of eccentric viewing or fixation with time. We scheduled testing of the low-vision patients with the Catford drum at $60 \mathrm{~cm}$ during the initial visit and on subsequent visits after one and six months of using standard low-vision aids. This consisted primarily of using high plus lenses from +5 dioptres to +32 dioptres based on the level of vision, along with basic instruction in low-vision principles. Lowvision aids are typically made for use with the eye that has the better visual acuity. Therefore testing of visual performance and the ability to learn eccentric viewing were evaluated with the patient's better eye, since there would be no training of the eye with the lower vision. Most patients in this clinic suffered loss of vision due to macular disease, usually secondary to age related macular degeneration and its sequelae. Patients who did not have any visual loss due in part to macular disease were excluded from this part of the study, since we were attempting to assess the ability to use eccentric viewing or parafoveal retinal areas for vision. A total of 15 patients were seen on follow-up visits in the initial testing, all one month after initial low-vision instruction.

\section{Results}

Our findings based on 82 eyes indicated that visual acuities as measured by the Catford drum significantly overestimate the subjective acuities as taken by the Snellen chart. This overestimation occurred in all but eight eyes with $90 \cdot 2 \%$, or 74 out of 82 eyes tested, giving this result. $6 \cdot 1 \%$ had equal visual acuities, and in $3.7 \%$ there was an underestimation of the subjective visual acuity by the Catford drum (Fig. 2). Therefore the great majority of the Snellen visual acuities recorded from $20 / 30$ to $20 / 400$ were overestimated by a factor ranging from 1.05 to 20.0 , average 4.73 . The most frequent overestimations of the Snellen visual acuity were by factors of 5 and 10 , which occurred in $36 \%$ of eyes tested (Fig. 3). Factors were calculated from the visual acuity fractions, being converted to decimal equivalents and then compared with each other. Decimal notation is 


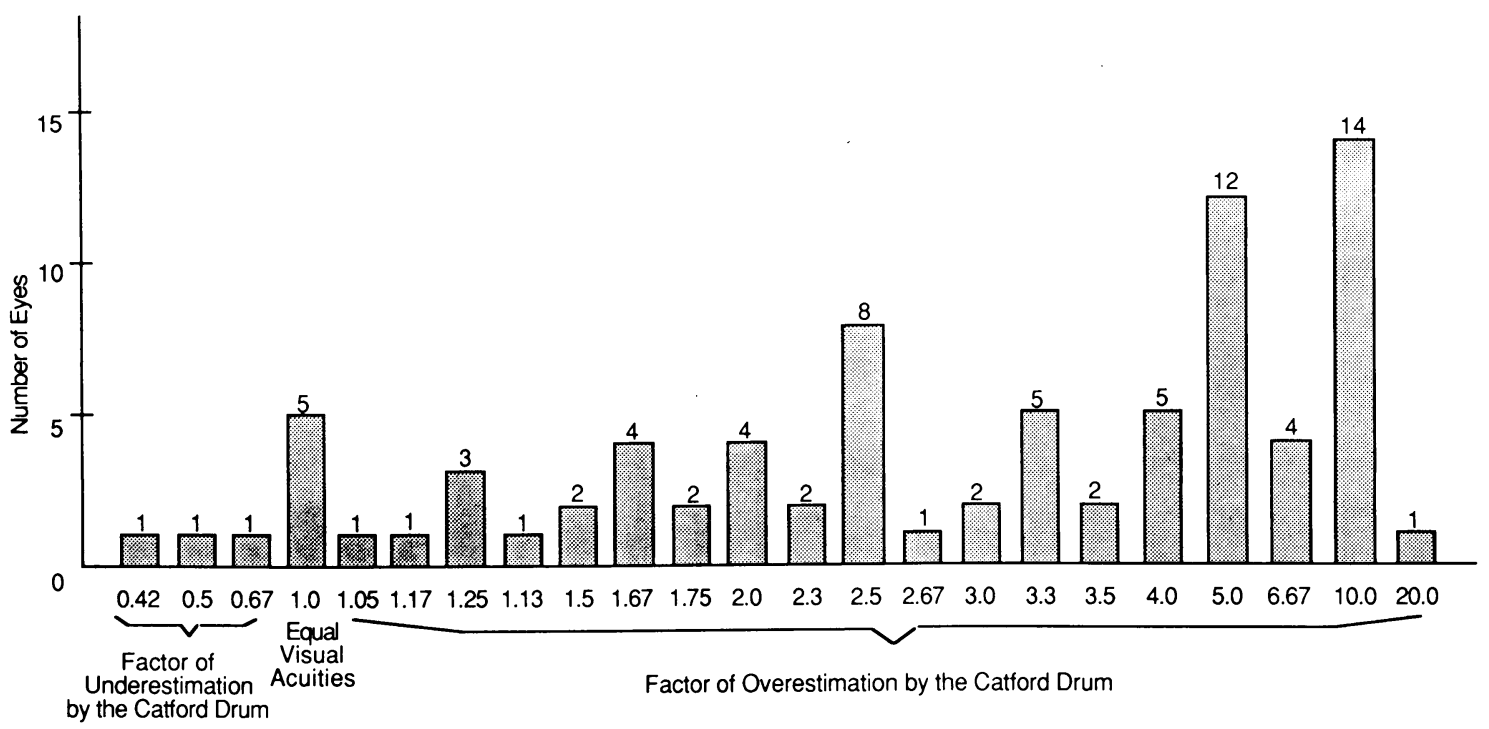

Fig. 3 Comparison of the Catford drum acuity with the Snellen acuity in each eye is done to obtain a factor of overestimation, underestimation, or equal visual acuity for each eye tested. The number of eyes with similar results is shown.

obtained by dividing the numerator by the denominator, and it is not a measure of the percentage of remaining vision or loss of vision. For example, 20/20 equals 1.0 and is an improvement by a factor of 5 over $20 / 100$ with its decimal equivalent of $0 \cdot 2$. The calculated correlation coefficient for the study was $+0 \cdot 40$.

In assessing visual performance as measured by the Catford drum in our low-vision patients we noted a considerable increase in Catford acuity, while the Snellen acuity remained without significant change during return visits. The results in 15 patients who were tested on follow-up visits one month after their initial low-vision instruction revealed that 12 out of the 15 patients $(80.0 \%)$ showed an improvement in Catford acuity. Three of the patients tested showed no change in their Catford acuity after one month (Fig. 4). This improvement in Catford visual acuity was by a factor ranging from $0 \cdot 3$ to $10 \cdot 0$, average $4 \cdot 08$. Thirteen of the 15 patients showed no change in their visual acuity on repeat testing with the Snellen chart, with one patient improving one line and the other dropping one line on the Snellen chart. No data are yet available from longer term follow-up patients.

\section{Discussion}

The results of our study indicate that the Catford acuities almost uniformly overestimate those taken by the Snellen chart. Probably several factors explain this finding. Part of the reason for the overestimation of Snellen visual acuities may be the calibration of the target sizes by Catford and Oliver. The diameter of the dot on the Catford drum was given a visual acuity which correlated with the overall size of the Snellen letter, with its designated visual acuity fraction. This was based on the fact that both the Catford dot and the Snellen letter subtend an equal angle at their viewed distance. Other investigators ${ }^{8}$ have pointed out the necessity of distinguishing between the 'minimum observable' object and the 'minimum separable' object. It is thought that the significant dimension of a Snellen letter for determining visual acuity is more likely the stroke width, which is $1 / 5$ of the Snellen letter height. If this is accepted, it would result in an overestimation of the Snellen visual acuities by a factor of 5 . An explanation for the poor correlation of results could in part be due to observer inaccuracy. Although the visual acuity determinations using the Catford drum were performed as Catford originally described, the ability to determine when an optokinetic nystagmus is present versus random eye movements may not always be accurately assessed by means of only visual methods of detection. However, other factors must be involved, since Khan et al.' used electrical means of recording eye movements but still produced only a +0.61 correlation coefficient.

Our data result in a poor correlation between objectve and subjective visual acuities, which differs from the results of Catford and Oliver. However, this finding is supported by the two other studies which have investigated the Catford drum, as mentioned 


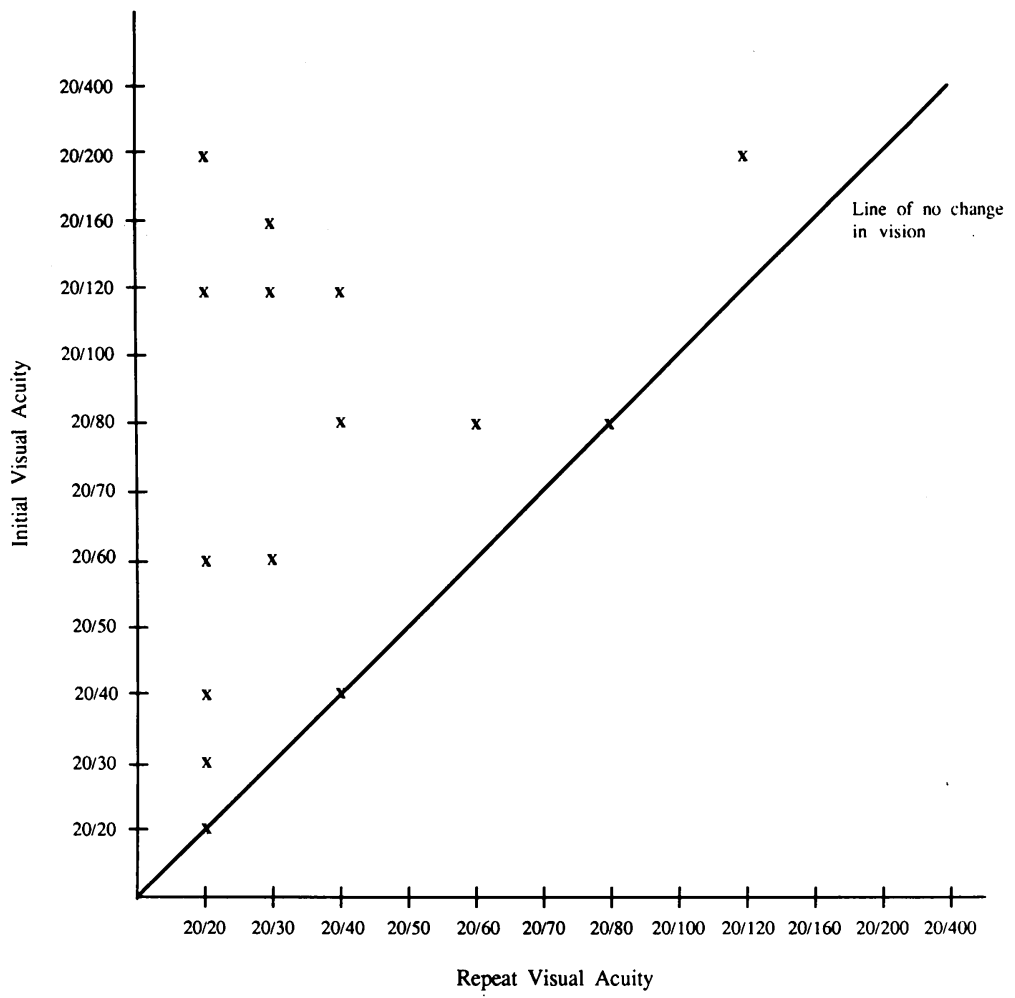

Fig. 4 Results of the Catford drum acuity on initial testing versus the Catford drum acuity obtained on one month follow-up visits. Each $\mathbf{x}$ represents one eye, with a total of 15 eyes tested.

earlier. The degree of overestimation by the Catford drum was noted to vary greatly with the level of vision being tested, and it is doubtful whether a recalibration of the Catford drum will be possible owing to the poor correlation of the data collected so far. For example, one patient had a Snellen acuity of $20 / 400$ but had a 20/20 result with the Catford drum, while another patient had a Snellen acuity of 20/30 and a Catford acuity of 20/20.

Finally, it is not clear how the ability to pick out moving black dots of different specific sizes which in theory are supposed to be equivalent to a corresponding Snellen letter correlates as a test of resolution. It would seem other factors must be involved, such as contrast sensitivity, motion detection and others.

In the second part of the study another method of visual acuity testing, the Catford drum, was used to test our theory that low-vision patients develop new skills that allow them to perform better than expected from traditional visual acuity measurements. The results show that the Snellen visual acuities did remain stable, as expected, in contrast to the Catford acuities, which improved on follow-up visits. Since this increase in Catford acuity cannot be explained on the basis of an improvement in the underlying disease, other processes must be involved, such as learning to use eccentric areas for vision.

This conclusion is given support by a recent report from Harris et al. ${ }^{9}$ at the Wilmer Ophthalmological Institute. They report that patients with macular disease do not spontaneously use their best eccentric area of retina for vision. Furthermore, they report $90 \%$ of their patients were able to show a two- to four-fold improvement in visual acuity when eccentric or parafoveal areas were specifically tested. They conclude: 'training of fixation behaviour to favour eccentric areas of retina ... may prove valuable in visual rehabilitation.' This is a view we have shared in our clinic for a long time and for which the above data lend further support in our opinion. The time course during which the improvement takes place suggests there is an 'adaptation or learning phenomenon' occurring.

A major factor in this adaptation process might be the fact that our patients are becoming more skilful in the use of eccentric or parafoveal retinal areas with the help of low-vision aids and instruction. This is compatible with the fact that we have noticed that in patients with very large central scotomas the improvement in performance is less and takes longer than in patients with smaller central scotomas. In our 
study three of the 15 patients seen on their initial follow-up after one month of training showed no improvement in the Catford visual acuity. These results might be explained by the extent of the patients' macular disease. If they had developed a larger area of macular involvement this would result in a larger central scotoma. Therefore one could theorise that these patients might require more practice and skills to be able to locate and develop the use of these parafoveal areas found at greater distances from the fovea. It would be interesting to review the fundus findings and compare visual fields in these patients to determine if larger central scotomas were present. In addition other factors almost certainly play an important part in the ability and rate of learning to use parafoveal retinal areas. For example, the patient's psychological state, influenced by the loss of a loved one or denial of their eye disease, can significantly delay this learning process from our past experiences with these patients. We expect these three patients will most likely show improvement with time. We await further follow-up visits on these patients and others for further confirmation of our initial findings

In summary, we consider most low-vision patients function at a higher level than would be predicted from their Snellen visual acuity. This seems to be based on their ability to learn the use of eccentric viewing, in other words their ability to use a parafoveal retinal area which has a better capacity for useful vision than their damaged fovea. Our current methods of assessing low-vision patients with the Snellen chart are inadequate. $67 \%$ or 55 out of 82 eyes tested had a visual acuity of $20 / 100$ or less. This makes the Snellen chart a poor choice for examining low-vision patients owing to its unreliability and lack of accuracy at that level of vision. It is obvious that other techniques must be tried, such as the 'full field E' visual acuity test of Harris et al., ${ }^{9}$ the Snellen chart at 5 feet $(1.5 \mathrm{~m})$, etc. The Catford drum may be a starting point in helping to assess better the visual performance and the true visual potential of lowvision patients.

We are continuing our study and will report further data later, but we hope this report will enable our fellow ophthalmologists to use this information to their patients' benefit and to initiate similar studies to test the validity of our observations.

\section{References}

1 Pearson RM. The objective determination of vision and visual acuity. BrJ Physiol Optics 1966; 23: 707-28.

2 Wolin LR, Dillman A. Objective measurement of visual acuity. Arch Ophthalmol 1964; 71: 822-6.

3 Millodot M, Miller D, Jernigan ME. Evaluation of an objective acuity device. Arch Ophthalmol 1973; 90: 449-52.

4 Catford GV, Oliver A. A method of visual acuity detection. Orthoptics-Proceedings of the Second International Orthoptic Congress. Amsterdam: Excerpta Medica, 1971: 183-7.

5 Catford GV, Oliver A. Development of visual acuity. Arch Dis Child 1973; 48: 47-50.

6 Atkinson J, Braddick O, Pimm Smith E, Ayling L, Sawyer R. Does the Catford drum give an accurate assessment of acuity? Br J Ophthalmol 1981; 65: 652-6.

7 Khan S, Chen KF, Frenkel M. Subjective and objective visual acuity testing techniques. Arch Ophthalmol 1976; 94: 2086-91.

8 Sheridan M. The STYCAR graded-balls vision test. Dev Med Child Neurol 1973; 15: 423-32.

9 Harris M, Robins D, Dieter J, Fine S, Guyton D. Eccentric visual acuity in patients with macular disease. Ophthalmology 1985; 92 : 1550-3.

Accepted for publication 28 October 1986. 